1st Step. Exactly as in [1], we prove that $(\operatorname{Pr} . \mathrm{AP})_{n}$ has a weak solution $u^{n}$ for which the inequality (1) holds.

$2 n d$ Step. Following the argument in [1] with a slight modification we show that it is possible to select a subsequence $\left\{v^{n}\right\}$ of $\left\{u^{n}\right\}$ such that $v^{n}$ converges to $\bar{u}$ weakly in $\hat{H}_{\sigma}^{1}(\hat{B}), v^{n} \mid E$ converges to 0 strongly in $L_{2}(E)$, and that for every compact set $K \subset \hat{\Omega}$ the restriction of $v^{n}$ to $K$ converges strongly in $L_{2}(K)$. It is easy to verify that $u=\vec{u} \mid \hat{\Omega}$ satisfies (2).

3rd Step. Use the following lemma to show that $u \in \hat{H}_{\sigma}^{1}(\hat{\Omega})$. We recall (A3).

LemmA 5. Let $w \in \hat{H}_{\sigma}^{1}(\hat{B})$. If $w=0$ in $E=\hat{B}-\hat{\Omega}$, then $w \mid \hat{\Omega} \in \hat{H}_{\sigma}^{1}(\hat{\Omega})$.

4 th Step. Following the argument in [2] or reexamining the procedure in the 2 nd step, we realize that $u(t)$ satisfies the second condition of Definition 3 after possible redefinition on a null set of $t$.

\title{
REFERENCES
}

1. E. Hopf, Über die Anfangswertaufgabe für die hydrodynamischen Grundgleichungen, Math. Nachr. 4 (1951), 213-231.

2. J. Serrin, Initial value problem for Navier-Stokes equations, nonlinear problems, Univ. of Wisconsin Press, Madison, 1963, pp. 69-98.

The University of Tokyo, Japan and

National Research Institute for Mathematical Sciences, Pretoria, SOUTH AFrICA

\section{ERRATUM, VOLUME 74}

C. A. Berger and L. A. Coburn, $C^{*}$-algebras of translations and multipliers, pp. 1008-1012.

The proof of Lemma 2 is incorrect. Theorem 1 remains correct provided we add the hypothesis that $G$ has an element which acts ergodically by translation. In this case, we can apply the pointwise ergodic theorem and the Lebesgue dominated convergence theorem in place of Lemma 2. 